Simon: Ueber Verbindungen des Chlorlithiums etc. 371

\title{
Ueber die Verbindungen des Chlorlithiums und des Chlormagnesiums mit Alkoholen;
}

ron

\section{S. E. Simon.}

Graham ${ }^{1}$ ) hat zuerst (1827) durch Einwirkung ron Alkohol auf wasserfreie Chloride krystallisirte Verbindungen erhalten, in welchen er eine durch ein einfaches Aequivalentrerhältniss sich ausdrückende Quantität von Alkohol ermittelt und deshalb diese Körper als chemische Verbindungen des Alkohols mit jenen Chlormetallen bezeichnet hat.

Das Salz, welches er zuerst mit dem Alkohol verbunden, war das so leicht zerfliessliche Chlorcalcium, welches sich bekanntlich auch mit Wasser vereinigt und in Verbindung mit demselben krystallisirt. Er stellte ferner Verbindungen dar vou Chlormangan und Chlorzink, sowie auch von salpetersaurer Magnesia und salpetersaurer Kalkerde mit dem Alkohol.

Der Alkohol vertritt nach Graham's Auffassung in den von ihm dargestellten Verbindungen (von ihm Alkoholate genannt) die Stelle des Krystallwassers.

Die Existenz dieser Verbindungen wurde von Einbrodt ${ }^{2}$ ) bezweifelt. Derselbe untersuchte die durch Einwirkung von Alkohol auf wasserfreies Magnesiumnitrat entstehenden Verbindungen, fand die Zusammensetzung nicht constant und glaubte nach diesen seinen Beobachtungen das Bestehen der Graham'schen Alkoholverbindungen, welches bereits früher von Berzelius ${ }^{3}$ ) als nicht ganz sicher angesehen worden ist, in Zweifel ziehen zu müssen. Diese Zweifcl wurden indessen durch Chodnew's $\left.{ }^{4}\right)$ Bcobachtungen im Wesentlichen gehoben, und es wurde die Auffassung von Graham bezüglich des Bestehens bestimmter derartiger Verbindungen durch

1) Pogg. Ann. 15, 150.

2) Ann. Chern. Pharm. 65, 115.

3) Berz. Jahresber. 1828, S. 259.

4) Ann. Chim Phys. 71, 241. 


\section{Simon: Ueber Verbindungen des Chlorlithiuns}

die bald darauf folgende Auffindung anderer derartiger Alkoholate bestätigt. Die betreffenden Arbeiten sind von Levy ${ }^{1}$ ), Robiquet ${ }^{2}$, de Luynes ${ }^{3}$, und Bauer ${ }^{4}$ ) ausgeführt worden.

Ueberblickt man die Reihe der bisher dargestellten Verbindungen von Alkohol mit Salzen, so stellt es sich heraus, dass jene Salze zu der Klasse der in Wasser leicht löslichen und zugleich mit Krystallwasser sich vereinigenden gehören.

In Anbetracht dieses Umstandes habe ich bei meiner Untersuchung das Augenmerk auf diese Gruppe von Salzen gerichtet und dabei Resultate erzielt, welche dazu beitragen, den Satz zu verallgemeinern, dass solche Salze an Stelle des Krystallwassers Alkohole aufzunehmen im Stande sind.

Die von mir dargestellten, nachstehend beschriebenen Alkoholate, welche ich als das vorläufige Resultat meiner Untersuchmngen bezeichne, sind die Verbindungen des Chlorlithiums und Chlormagnesiums mit Aethyl- und Methy]alkohol.

\section{Alkoholate des Chlorlithiums.}

Von den Chloriden der Alkalien ist das Chlorlithium das am leichtesten zerfliessliche und in Wasser leichtest lösliche Chlorid, welches auch bekanntlich Hydratwasser aufzunehmen im Stande ist. Es entfernt sich in dieser Beziehung namhaft von dem Chlorkalium, Chlornatrium und Chlorcäsium; ihm nähert sich das Chlorrubidium.

Die Darstellung des reinen wasserfreien Chlorlithiums hat seine Schwierigkeit. Ich fand folgenden Weg für diesen Zweck recht geeignet: Kohlensaures Lithium wird in Salzsäure gelöst, die Flüssigkeit mit etwas Ammoniak versetzt, um geringe Mengen des darin oft enthaltenen Eisenoxyds abzuscheiden, und die Lösung nun mit so viel reinem Sal-

1) Ann. Chim. Phys. [3] 16, 309.

2) Lieb. Jahresber. 1854, S. 559 .

3) Dies. Journ. 80, 503.

4) Daselbst 80, 361 . 
miak vermischt, dass etwa gleiche Aequivalente beider Salze sich in Lösung befinden. Die filtrirte Lösung wird nun zur Trockne eingedampft, das erhaltene Salz dann zur Beseitigung des Krystallwassers von Chlorlithium während einiger Zeit in einem Luftbade auf eine $100^{\circ}$ etwas übersteigende Temperatur erhitzt und nach völligem Austrocknen in eine bedeckte Platinschale gebracht. Diese wird alsdann durch eine kräftige Gasflamme (zweckmässig ist ein Gasgebläse) langsam zum Glühen gebracht, wobei der Salmiak entweicht und das Chlorlithium wasserfrei zurückbleibt. Dieser Weg empfiehlt sich aus dem Grunde, weil durch Erhitzen des wasserhaltigen Chlorids, ähnlich wie beim wasserhaltigen Chlormagnesium, das Hydratwasser in der Hitze das Chlorid zerlegt.

Das mit diesen Vorsichtsmaassregeln dargestellte Chlorlithium ist eine leicht schmelzbare Masse, die nach dem Erkalten einen krystallinisch blättrigen, in Wasser völlig und leicht löslichen Kuchen bildet. - Mit diesem Materiale wurde zunächst das

\section{Aethylalkoholat}

in folgender Weise bereitet:

Reiner, durch Destillation mit Natriummetall von den letzten Spuren anhaftenden Wassers befreiter Aethylalkohol wird in ein weites, durch einen weichen Kork luftdicht abgeschlossenes Reagenzglas gebracht, und in den Alkohol das in kleine Stücke zertheilte Chlorid nach und nach eingetragen.

Die Vereinigung beider Körper erfolgt unter so starker Erwärmung, dass auf eine Abkühlung mit Wasser Bedacht genommen werden muss. Man fügt das Chlorid dem Alkoholate in solchen Mengen zu, als bei der Siedetemperatur der Lösung davon aufgenommen wird.

Die so erhaltene, etwas durch Lithiumoxyd getrübte Flüssigkeit lässt man in der Wärme durch Absetzen sich klären und sie sodann (am besten in einem grossen Wasserbade) langsam erkalten. Wegen der Löslichkeit des Alkoholats in überschüssigem Alkohol ist man meistens benöthigt, 


\section{Simon: Ueber Verbindungen des Chlorlithiums}

die für die Krystallisation erforderliche Abkühlung durch Eis oder eine Kältemischung zu bewirken. - Durch diese Procedur erhält man in der abgeklärten Flüssigkeit Krystallkrusten des Alkoholats. Um dieselben zu isoliren, giesst man die trübe Mutterlauge ab, schabt die feste Masse aus dem Glase heraus, presst sie schnell mit Fliesspapier ab und bringt sie dann in ein gut verschliessbares Glas.

Die so dargestellte Verbindung bildet farblose, durchsichtige, prismatische, fettglänzende Krystallaggregate. Dieselben sind sehr leicht in Wasser und Alkohol löslich. Partikel derselben auf Wasser gebracht, schwimmen auf der Oberflüche und gerathen, indem sie sich lösen, in heftig drehende Bewegung.

Die Substanz hat den adstringirenden Geschmack des Chlorlithiums.

Die Analyse dieses Körpers wurde auf zwei verschiedene und zu dem gleichen Resultate führende Weisen ausgeführt: einerseits durch Erhitzen einer gewogenen Quantitüt der Substanz bis zur Verflïchtigung des gebundenen Alkohols, andererseits durch Ermittelung des Chlorgehalts einer gekannten Menge der gedachten Verbindung.

Hierbei ergaben sich folgende Resultate:

1.479 Grm. Substanz hinterliessen 0,276 Grm. trocknes Chlorlithium, entsprechend $15,60 \%$ Chlor.

1,440 Grm. Substanz hinterliessen 0,273 Grm. trocknes Chlorlithium, entsprechend $15,88 \%$ Chlor.

Bei der zweiten Art der Analyse, wobei das Chlor dureh Silberlosing bestimmt wurde, ergaben:

1,361 Grm. Substanz 0,858 (trm. Chlorsilber, entsprechend 15,59 pCt. Chlor.

Hieraus berechnet sich die atomistische Zusammensetzung $\mathrm{Li} C \mathrm{Cl}$ $+4\left(\mathrm{C}_{2} \mathrm{H}_{5} \mathrm{OH}\right)$, welche Verbindung $15,67 \%$ Chlor erfordert.

Dieses Alkoholat enthält die gleichen Atome Aethylalkohol, als das Hydrat Wasseratome enthält.

\section{Chlorlithiummethylat.}

Der Methylalkohol verbindet sich, wie der Aethylalkohol, unter starker Erwärmung mit dem Chlorlithium und bildet 
damit eine krystallisirbare Verbindung. Die Reindarstellung derselben bietet aber ungleich grössere Schwierigkeiten, als die Darstellung der entsprechenden Aethylverbindung.

Zur Gewinnung derselben wird in sorgfältig durch Destillation mit metallischem Natrium entwässerten Methylalkohol das Chlorlithium wiederum in Gestalt kleiner Stückchen eingetragen, wobei die Vereinigung unter starker Erhitzung erfolgt. Die gesättigte, etwas festes Chlorlithium enthaltende Flüssigkeit wird nach klarem Abgiessen auf - $15^{\circ}$ bis $16^{\circ}$ erkaltet, wobei sich nun erst Krystalle bilden, die in der Mutterlauge leicht zerfliessen und bei gewöhnlicher Temperatur schnell schmelzen. Um sie trocken darzustellen, giesst man die Mutterlauge von den Krystallen $a b$ und presst letztere schnell zwischen Fliesspapier.

Die so bereitete krystallinische Masse ist höchst zerHiesslich und sehr leicht schmelzbar, so dass sie bei warmer Lufttemperatur sogar eine Flüssigkeit bildet.

Ihre Zusammensetzung wurde durch Ermittelung des Chlorgehaltes bestimmt, wobei sich folgendes Resultat ergab:

2,167 Grm. Substanz lieferten 2,264 Grm. Chlorsilber, entsprechend $25,82 \%$ Chlor.

1,222 Grm. Substanz lieferten 1,281 Grm. Chlorsilber, entsprechend $25,91 \%$ Chlor.

Hieraus ergicbt sich die Formel: $\mathrm{LiCl}+3\left(\mathrm{CH}_{3} \mathrm{OH}\right)$, nach welcher die Menge des Chlorgehaltes sich auf $25,63 \%$ beziffert.

\section{Chlormagnesinmalkoholat.}

Das für diese und die folgende Verbindung erforderliche wasserfreie Chlormagnesium wurde durch Glühen des völlig getrockneten Doppelsalzes von Chlormagnesium und Salmiak hergestellt.

Obschon bei dieser Procedur alle Vorsichtsmaassregeln beachtet worden sind, konnte doch nur ein Schmelzkuchen des Chlorids erhalten werden, welcher zwar nicht erhebliche, aber doch immerhin wahrnehmbare Mengen von Magnesia (in Alkohol unlöslich) hinterliess.

Bei der Herstellung dieser Verbindung wurde im Uebrigen in analoger Weise, wie bei der Lithiumverbindung, ver- 


\section{Simon: Ueber Verbindungen des Chlorlithiums}

fahren. In reinem wasserfreien Alkohol wurde das zerstückelte Chlormagnesium portionsweise eingetragen, und die dabei auftretende starke Erwärmung durch Abkühlung vermindert. $Z u$ hohe Temperatur nämlich bewirkt leicht, wie auch bei der Lithiumverbindung, eine Zersetzung unter Abscheidung von Oxyd. Die bei mässiger Erwärmung noch einen unlöslichen Bodensatz von Chlormagnesium enthaltende Flüssigkeit wird durch Absetzenlassen von der stets sich aussondernden Magnesia befreit und sodann, etwa durch Wasser, auf die Lufttemperatur erkaltet.

Am Rande des Gefässes entstehen dann fett glänzende Krystallaggregate, die im Wasser sich leicht lösen und an der Luft schnell zerfliessen. Auch sie gerathen, auf Wasser gebracht, in die oben erwähnte kreisende Bewegung. Im Uebrigen zeigt dieses Salz den Charakter des bekanntlich leicht zerfliesslichen Chlormagnesiums.

Die Analyse wurde durch Ermittlung der Menge des Chlorsilbers ausgeführt, welche sich aus der Lösung einer bekannten Menge des Alkoholats mit Silbernitrat abgeschieden hatte. Dabci ergaben sich folgende Zahlenwerthe:

1,149 Grm. Substanz lieferten 0,885 Grm. Chlorsilber, entsprechend $19,04 \%$ Chlor, 0,339 Grm. pyrophosphorsaure Magnesia, entsprechend 6,38\% Magnesium.

0,941 Grm. Substanz lieferten 0,710 Grm. Chlorsilber, entsprechend 18,68\% Chlor, 0,277 Grm. pyrophosphorsaure Magnesia, entsprechend $6,36 \%$ Magnesium.

Jemuach berechnet sich die Formel:

$$
\mathrm{Mg} \mathrm{Cl}_{2}+6\left(\mathrm{C}_{2} \mathrm{H}_{5} \mathrm{OH}\right) \text {. }
$$

Jiese Verbindung erfordert:

$$
\begin{array}{lr}
\text { Chlor } & 19,13 \\
\text { Magnesium } & 6,46
\end{array}
$$

\section{Ohlormagnesiummethylat.}

Der Darstellung des Chlormagnesiummethylats treten, wie auch der Bereitung des Chlorlithiummethylats, entschieden grössere Schwierigkeiten, als der Herstellung der entsprechenden Aethylate entgegen. Dieselben beruhen in der geringeren Neigung der Methylate, zu krystallisiren und auf diese Weise sich von den Mutterlaugen zu isoliren. Eine 
und des Chlormagnesiums mit Alkoholen. 377

andere Schwierigkeit hat ihren Grund in der leichten Zersetzbarkeit dieser Mothylate. Bei denselben macht sich nämlich durch Erwärmen eine Einwirkung des Alkohols auf das Chlorid geltend, wobei sich unter Abscheidung ron Oxyd Chlormethyl bildet. Bei Vermeidung zu starker Erwärmung indessen kann man diesen Nebenprocess doch derart einschränken, dass die bezügliche Verbindung in Aggregaten, wenn auch von kleinen Krystallen, darstellbar ist, die sich nun durch Abpressen mit Fliesspapier isoliren lassen.

Auf diese Weise erhielt ich das Magnesiummethylat als eine in Blättern krystallisirende, fettglänzende, weiche und sehr zerfliessliche Masse, deren Zusammensetzung durch Ermittelung ihres Chlorgehaltes, so wie ihres Gehaltes an Magnesia, festgestellt worden ist.

Dabei ergaben sich folgende Zahlenwerthe:

$0,481 \mathrm{Grm}$. Substanz lieferten 0,465 Grm. Chlorsilber, entsprechend 23,92\% Chlor, 0,185 Grm. pyrophosphorsaure Magnesia, entsprechend 8,30\% Magnesium.

0,329 Grm. Substanz lieferten 0,319 Grm. Chlorsilber, entsprechend 23,97\% Chlor, 0,124 Grm. pyrophosphorsaure Magnesia, entsprechend 8,16\% Magnesium.

Hieraus ergiebt sich annäherungsweise die Zusammensetzung:

$$
\mathrm{Mg} \mathrm{Cl}_{2}+6\left(\mathrm{CH}_{3} \mathrm{OH}\right)
$$

welche Verbindung erfordert:

$$
\begin{array}{lc}
\text { Chlor } & 24,73 \% \\
\text { Magnesium } & 8,36 \%
\end{array}
$$

Ausser den oben beschriebenen Chloridalkoholaten habe ich auch noch Alkoholate von Bromverbindungen, so vom Bromcadmium, ferner Alkoholate der Chloride von Schwermetallen, wie des Chlorzinks und Chlorkupfers, auch deren Bromide, hergestellt.

Die Beschreibung dieser Verbindungen werde ich mit der Darlegung der Resultate einer Untersuchung über die Alkoholverbindungen von salpetersauren Salzen mittheilen, welche bis jetzt noch nicht zum Abschluss gekommen ist.

Berlin, im Juni 1879. 